\title{
Predetermined Time Constant Approximation Method for Optimising Search Space Boundary by Standard Genetic Algorithm
}

\author{
Kumaran Rajarathianm, J. Barry Gomm, Karl O. Jones, DingLi Yu
}

\begin{abstract}
In this paper, a new predetermined time constant approximation $\left(T s_{p}\right)$ method for optimising the search space boundaries to improve SGAs convergence is proposed. This method is demonstrated on parameter identification of higher order models. Using the dynamic response period and desired settling time of the transfer function coefficients offered a better suggestion for initial $T s_{p}$ values. Furthermore, an extension on boundaries derived from the initial $T s_{p}$ values and the consecutive execution, brought the elite groups within feasible boundary regions for better exploration. This enhanced the process of locating of the optimal values of coefficients for the transfer function. The $T s_{p}$ method is investigated on two processes; excess oxygen and a third order continuous model with and without random disturbance. The simulation results assured the $T s_{p}$ method's effectiveness and flexibility in assisting SGAs to locate optimal transfer function coefficients.
\end{abstract}

Key words: Predetermined Time Constant Approximation; Genetic Algorithms; Search Space Boundary Constraints; Premature Convergence.

\section{INTRODUCTION}

Search space boundary constraint is one of the common phenomena that lead to premature convergence in standard genetic algorithms (SGAs). An optimisation process has prematurely converged to a local optimum if it is no longer able to explore other parts of the search space region than the area currently being explored and there exists another region that may contain a superior solution [1]. This is especially true when the optimum values are located near to the boundary region or outside the boundary region. Therefore, an optimum search space region is required for better exploration and to avoid premature convergence. Parameter identification of continuous higher order models where the model parameters distinguish the dynamic characteristics of system are of particular concern. Without any prior knowledge of the transfer function coefficients, it is highly infeasible to predict the search space upper ( $\left.S B_{\text {Upper }}\right)$ and lower boundaries $\left(S B_{\text {Lower }}\right)$.

Significant work has been undertaken to improve the defined search space to an optimal solution. Based on the complex Box technique, a boundary search method for optimisation problems in the case of the optimal solution at the boundary was proposed [2]. A technique for resolving the structural optimisation difficulties in quantising the subjective uncertainties of active constraints are proposed by fuzzy logic formulation [3]. A new approach called the self-adaptive boundary search strategy for penalty factor selection within SGA was proposed [4]. This approach guides the SGA to preserve around constraint boundaries and improves the efficiency of attaining the optimal or near optimal solution.

Another method to improve the prematurity and to sustain the diversity population was proposed by Niche Genetic Algorithm (NGM) associated with isolation mechanism [5]. A comparison study was done on NGM and Annealing Genetic Algorithm (AGA) where the AGA has better premature convergence [6], however it is time consuming. Another method, named Accelerating Genetic Algorithm (AGM) was proposed to resizing the feasible region into the elite individual's adjacent region for better local searching and 
convergence [7]. Search space boundary reduction for the candidate diameter for each link by pipe index vector and critical path method along with modified genetic operator's derivatives was proposed [8][9]. Further, an improved AGM based on the saddle distribution by which adding random individuals into initial population to increase the searching ability of optimal solution was proposed [10]. Literature survey discloses that most techniques are considered based on limited or confined search space boundaries and involves complex mathematics. Also, the discussed research information has an initial approach about the search parameter and inevitably is time consuming for convergence.

This paper introduces and investigates the predetermined time constant approximation $\left(T s_{p}\right)$ method to improve the SGAs exploration and exploitation towards global optima. This method employs a novel search space boundary extension technique by $T s_{p}$ which guides the search to concentrate on optimal value within the boundaries of the feasible region of the solution space. The structure of this paper is as follows; first, 3 SGAs convergence states for an optimal value by search space boundary constraints are discussed. Second, the approximation process of predetermined time constant methods is discussed. Further, search space boundary extensions for better exploration and for optimal exploitation are discussed here. Third, the effectiveness of predetermined time constant approximation method is assessed with two processes.

\section{POLYNOMIAL COEFFICIENTS}

Consider a system can be modelled by the general order differential equation,

$$
a_{n} \frac{d^{n} y}{d t^{n}}+a_{n-1} \frac{d^{n-1} y}{d t^{n-1}}+\cdots+y=K_{p} f(t-\theta)
$$

where $f(t-\theta)$ is the input signal or forcing function with time delay, $y(t)$ is the output signal and $K_{p}$ is process gain. Assuming zero initial condition, $y(0)=0, y^{\prime}(0)=0$, and taking the laplace transform of equ. 1 gives the general order transfer function is of the form,

$$
G(s)=\frac{Y(s)}{F(s)}=\frac{K_{p}}{a_{n} s^{n}+a_{n-1} s^{n-1}+\cdots+a_{1} s+1} e^{-\theta s}
$$

where $a_{n} \ldots a_{1}$ are coefficients of the denominator polynomial. The denominator polynomial coefficients provide a foundation for determining a system's dynamic response characteristics. In particular the system's poles directly define the components in the homogeneous response. Thus, optimal poles identification is primarily considered here.

\section{CONVERGENCE CONSTRAINTS OF SGAs BY SEARCH SPACE BOUNDARIES}

In most situations, selecting the search space boundaries is delicate if there is no prior knowledge of optimum value location. Thus, randomly selected search space boundary is a significant factor which leads the SGAs are often converged and trapped in local optima, resulting suboptimal solutions. Particularly, it locates near the boundary or outside of boundary.

As shown in figure 1, the SGAs convergences by search space boundary constraints can be classified by three states;

- State 1 - If the optimal value $\left(X_{i}\right)$ located within uniformly distributed elite group around boundary region $\left[X_{i}-\Delta_{G O}, X_{i}+\Delta_{G O}\right]$, the genetic operators have higher probability of converging to global optimum. Thus, the randomly generated initial population within well distributed elite group search boundary has higher probability exploring and exploiting a better parent chromosome. Further, the selected parent chromosome will be evaluated by genetic precision process (selection, crossover and mutation) to produce fitter offspring without any convergence constraint.

- State 2 - If the $X_{i}$ located near ([SB $\left.B_{\text {Lower, }}, X_{i}-\Delta_{G O}\right]$, $\left[X_{i}+\Delta_{G O}, S B_{\text {Upper }}\right]$ ), the SGAs possibly will converge to local minima. The elite group which is distributed near the boundary may have located a part of elite group at outer boundary. If the elite group at outer part may have the genetic information of optimal value, the genetic operators 
will suffer to exploit the optimal value and the exploration process will retard. As a result, the search space boundary constrains will lead the SGAs to converge to local minima.

- State 3 - If the $X_{i}$ located outside the boundary region [S$B_{\text {Lower }}>X_{i}>S B_{\text {Upper }}$ ], the SGAs will fail to explore and exploit the optimal value. The simulation may retarded and stopped.

where $S B_{\text {Lower }}$ is lower search boundary, $S B_{\text {Upper }}$ is upper search boundary and $\Delta_{G O}$ is the genetic operator for convergence precision.

By approximating the

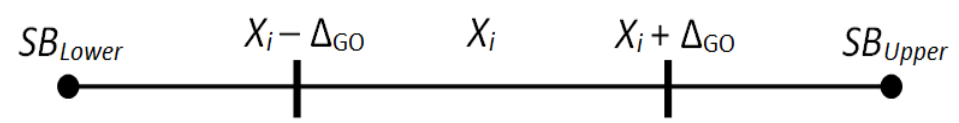

Figure 1: Schematic diagram of feasible serach space boundary region distribution of the elite group in a boundary region at the initial stage, gives the genetic operators opportunity to locate the optimal value rapidly without any constraint. To improve searching space boundaries for optimal model identification, a straightforward trial and error technique without a mathematical constraint is introduced here, named predetermined time constant approximation $\left(T_{S p}\right)$. The approximation process can be simplified as follows;

- Selecting $\sigma T_{s}$, where $\sigma$ is the settling band in \%. $(\sigma=3,4$ and 5$)$. The selection of desired $\sigma$ is according to the raggedness of dynamic response.

- Estimating process's dynamic response period $\left(D R_{P}\right)_{(\tau 2-\tau)}$. At $C(t)=O_{(T=\tau)}$ to $C(t)=1 \pm$ $\sigma(\%)_{(T=\tau 2)}$, where $C(t)$ is desired settling point.

- Approximating an initial $\tau_{1}=D R_{P(T 2-\tau)} / \sigma$.

- Calculating initial $T_{S p}$ by identified $T_{1}$ according to the respective transfer function coefficients $\left(a_{n} s^{n}+a_{n-1} s^{n-1}+\cdots+a_{1} s+1\right)$.

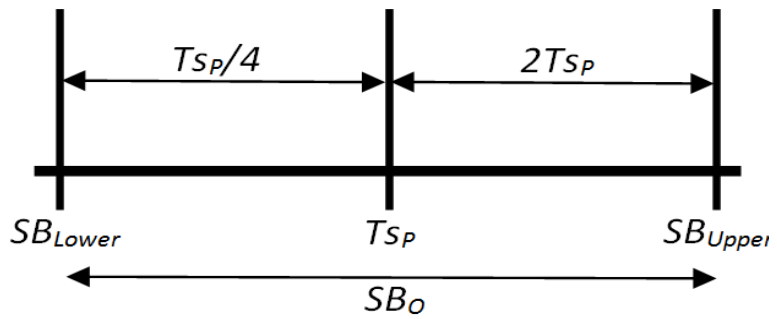

Figure 2: Optimising the search space boundary by $T s_{p}$

where $S B_{O}$ is optimum search space boundary, $S B_{\text {Lower }}$ is lower search boundary and $S B_{\text {Upper }}$ is upper search boundary. An optimum search space boundary can be approximated by $T_{S p}$, as illustrated in figure 2 and can be expressed as;

$$
S B_{O}=\left\{S B_{O} ; S B_{\text {Lower }} \leq T_{S_{P}} \leq S B_{\text {Upper }}\right\}
$$

For an $S B_{O}$, the $S B_{\text {Upper }}$ and $S B_{\text {Lower }}$ are extended by $100 \%$ and $75 \%$ from $T_{S p}$, respectively. Especially, $100 \%$ of extension for $S B_{\text {Upper }}$ is required as the optimal solution mostly located near to the upper boundary region. Such a search space extension is required for SGAs to explore the elite groups which are uniformly distributed within boundaries and to exploit the $X_{i}$.

\section{SIMULATION STUDIES}

To illustrate the non-complexity and effectiveness, the proposed $T s_{p}$ method is applied on two industrial processes; excess oxygen $\left(E \mathrm{O}_{2}\right)$ and $3^{\text {rd }}$ order transfer function.

\section{Process 1 - Excess Oxygen $\left(E_{2}\right)$}

A raw numerical data of $\mathrm{EO}_{2}$ is collected from a real industrial furnace by empirical technique for 1000 seconds with 5 seconds interval. As illustrated in fig. 3, the process response of $\mathrm{EO}_{2}$ is exhibiting the first-order plus dead-time (FOPDT) dynamic system. The data was gathered by the step input of increasing air ratio from 9.5 to 10.5 in volumetric. 
As discussed earlier, the time constant $\left(T_{S}\right)$ of transfer function are primarily considered here for an optimal model identification by $T_{S p}$ method. Whereas, the process gain $\left(K_{p}\right)$ and transport delay $(\theta)$ can be approximated by close observation on transient response. As illustrated on the transient response of $\mathrm{EO}_{2}$, the $K_{p} \approx 1.54$ and $\theta \approx 160$ s. As a result, an extension on the search space

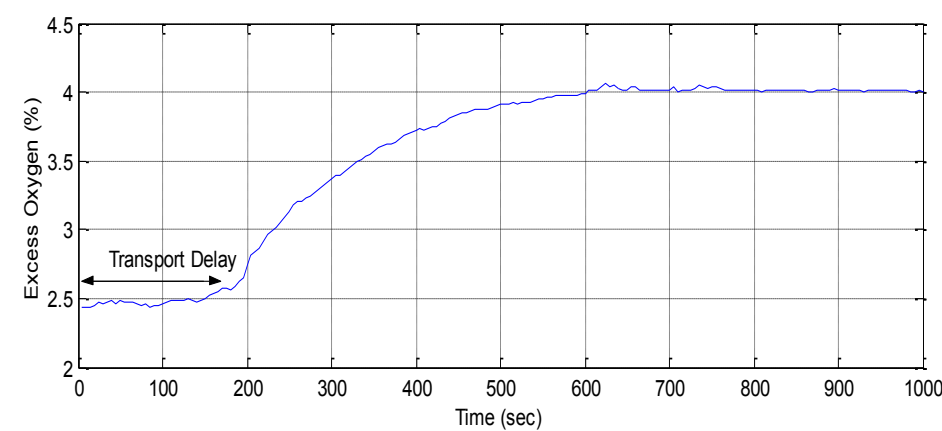

Figure 3: Step response of $\mathrm{EO}_{2}$ boundaries are approximated for $K_{p} \in[1: 2]$ and $\theta \in[50: 200]$.

According to the $\mathrm{EO}_{2}$ response, the $D R_{P(T 2-\tau)}=700 \mathrm{~s}-100 \mathrm{~s}=600 \mathrm{~s}$. Selecting $\sigma T_{s}=$ $5 \mathrm{~T}_{\mathrm{s}}$, as the desired $T_{s}$ is $1 \%$ settling band, gives the initial $T_{1}$ as $120 \mathrm{~s}$. For $\mathrm{EO}_{2}$, the selection of an optimal model is a $3^{\text {rd }}$ order transfer function. Therefore, the $T_{S p}$ for the $3^{\text {rd }}$ order polynomial coefficients can be approximated,

$$
\begin{aligned}
& \tau_{1} s=120 ;-\rightarrow\left(T_{1} s\right)^{3}+3\left(T_{1} s\right)^{2}+3 T_{1} s+1 \\
& =1.728 e^{6} s^{3}+4.32 e^{4} s^{2}+3.6 e^{2} s+1
\end{aligned}
$$

\section{Process $2-3^{\text {rd }}$ Order Transfer Function}

For simulation study, the transfer function of a $3^{\text {rd }}$ order process is selected with process gain $\left(K_{p}=10\right)$,

$$
G(s)=\frac{10}{15 s^{3}+78 s^{2}+6 s+1}
$$

The particular motive of selecting this $3^{\text {rd }}$ order transfer function is that it has a real pole at -5.1245 and a pair of complex poles at $-0.0378 \pm 0.1076 \mathrm{i}$ which are exhibiting a significant oscillatory response. Also, to assess the $T_{S p}$ method's flexibilities and effectiveness, the $3^{\text {rd }}$ order transfer function coefficients are moderately small parameters. So, an appropriate search space boundary extension is required.

According to the $3^{\text {rd }}$ order process step response (Fig. 3), the $D R_{P(T 2-\tau)}=123 \mathrm{~s}-0 \mathrm{~s}=$ 123s. Selecting $\sigma T_{s}=5 T_{s}$, as the desired $T_{s}$ is $1 \%$ settling band, gives the initial $T_{1}$ is 24.6s. Therefore, the $T_{S p}$ for the $3^{\text {rd }}$ order polynomial coefficients can be approximated by,

$$
\begin{aligned}
& \tau_{1} s=24.6 ;-\rightarrow\left(T_{1} s\right)^{3}+3\left(T_{1} s\right)^{2}+3 T_{1} s+1 \\
& =14887 s^{3}+1815.5 s^{2}+73.8 s+1
\end{aligned}
$$

\section{DISCUSSION}

Generally, the all process of search space boundary adjustment and an optimal $X_{i}$ identification can be simplified as follows;

1. Initial attempt - Identified $T_{S p}$ according to the respective transfer function coefficients are applied with $100 \%$ extension on $S B_{\text {Upper. }}$ The $S B_{\text {Lower }}$ is extended to approximately $95 \%$ (10) instead $75 \%$ for better exploration at beginning stage. Execute the SGAs.

2. Second attempt - Genetically identified $T_{S p}$ of respective transfer function coefficients by initial attempts are extended accordingly ( $S B_{\text {Upper }}$ to $100 \%$ and $S B_{\text {Lower }}$ to $75 \%$ ) to optimise $S B_{O}$. Execute the SGAs.

3. Subsequent attempt - Continuing the SGAs execution with unchanged boundary search approximation by second attempt, until optimal $X_{i}$ and minimum sum of square error (SSE) attained.

4. *Subsequent attempt - If the extended boundary in second attempt is not a $S B_{O}$, consecutive boundary adjustment is essential until $S B_{O}$ achieved. Then, continuing the SGAs execution until optimal $X_{i}$ and SSE attained. 


\section{Simulation Results of $\mathrm{EO}_{2}$}

As illustrated in table 1 , the SGAs is explored well the entire search space boundaries and exploited the elite group within boundary region $\left[X_{i}-\Delta_{G O}, X_{i}+\Delta_{G O}\right]$ for $T_{S p}$ values of $S^{2}$ and $S^{1}$ at initial attempt. This can be seen the consistency of the $T_{S p}$ values of $S^{2}$ and $S^{1}$ in further execution with readjusted boundaries at $2^{\text {nd }}$ attempt. This has enhanced the exploitation an optimal $X_{i}$ at each subsequent attempted by SGAs.

Table 1: Simulation Results of $\mathrm{EO}_{2}$ Executions

\begin{tabular}{|c|c|c|c|c|c|c|c|c|c|c|c|}
\hline \multirow{2}{*}{$\begin{array}{c}\text { Execution } \\
\text { Cycle }\end{array}$} & \multicolumn{2}{|c|}{$s^{3}$} & \multicolumn{2}{|c|}{$S^{2}$} & \multicolumn{2}{|c|}{$S^{1}$} & \multirow{2}{*}{$\begin{array}{l}T s_{p} \\
\left(S^{3}\right)\end{array}$} & \multirow{2}{*}{$\begin{array}{l}T s_{p} \\
\left(S^{2}\right)\end{array}$} & \multirow{2}{*}{$\begin{array}{l}T s_{p} \\
\left(S^{\prime}\right)\end{array}$} & \multirow{2}{*}{ SSE } & \multirow{2}{*}{ Gen } \\
\hline & $S B_{U}$ & $S B_{L}$ & $S B_{U}$ & $S B_{L}$ & $S B_{U}$ & $S B_{L}$ & & & & & \\
\hline 1 & $3.5 \mathrm{e} 6$ & 10 & $8.6 \mathrm{e} 4$ & 10 & $7.2 \mathrm{e} 2$ & 10 & 8088.2 & 10085 & 178.73 & 0.86796 & 70 \\
\hline 2 & $1.6 \mathrm{e} 4$ & $2 \mathrm{e} 3$ & $2 \mathrm{e} 4$ & $2 \mathrm{e} 3$ & $3.5 \mathrm{e} 2$ & 40 & 4039.7 & 14074 & 180.02 & 0.49128 & 20 \\
\hline 3 & $1.6 \mathrm{e} 4$ & $2 \mathrm{e} 3$ & $2 \mathrm{e} 4$ & $2 \mathrm{e} 3$ & $3.5 \mathrm{e} 2$ & 40 & 2699.7 & 13304 & 180.38 & 0.51873 & 40 \\
\hline 4 & $1.6 \mathrm{e} 4$ & $2 \mathrm{e} 3$ & $2 \mathrm{e} 4$ & $2 \mathrm{e} 3$ & $3.5 \mathrm{e} 2$ & 40 & 4875.7 & 14995 & 183.64 & 0.49413 & 40 \\
\hline 5 & $1.6 \mathrm{e} 4$ & $2 \mathrm{e} 3$ & $2 \mathrm{e} 4$ & $2 \mathrm{e} 3$ & $3.5 \mathrm{e} 2$ & 40 & 8187.7 & 14524 & 181.41 & 0.48654 & 20 \\
\hline 6 & $1.6 \mathrm{e} 4$ & $2 \mathrm{e} 3$ & $2 \mathrm{e} 4$ & $2 \mathrm{e} 3$ & $3.5 \mathrm{e} 2$ & 40 & 8079.1 & 16513 & 184.16 & 0.53421 & 35 \\
\hline 7 & $1.6 \mathrm{e} 4$ & $2 \mathrm{e} 3$ & $2 \mathrm{e} 4$ & $2 \mathrm{e} 3$ & $3.5 \mathrm{e} 2$ & 40 & 4330.5 & 14555 & 177.2 & 0.5109 & 90 \\
\hline 8 & $1.6 \mathrm{e} 4$ & $2 \mathrm{e} 3$ & $2 \mathrm{e} 4$ & $2 \mathrm{e} 3$ & $3.5 \mathrm{e} 2$ & 40 & 4137.2 & 15028 & 181.88 & 0.48758 & 22 \\
\hline 9 & $1.6 \mathrm{e} 4$ & $2 \mathrm{e} 3$ & $2 \mathrm{e} 4$ & $2 \mathrm{e} 3$ & $3.5 \mathrm{e} 2$ & 40 & 9903.9 & 16043 & 182.3 & 0.51771 & 80 \\
\hline
\end{tabular}

Based on initial attempt, the elite groups of $T_{S p}$ value of $S^{3}$ are uniformly distributed around $X_{i}-\Delta_{G O}$ region. As illustrated table 1 , the $T_{S p}$ value of $S^{3}$ is still continuously evolving within boundary $S B_{0}$ region at each execution. Therefore, further readjustment on $S B_{O}$ boundaries is not required as the elite groups are still within the boundary range (state 1) as discussed section 3. For $3^{\text {rd }}$ order model of $\mathrm{EO}_{2}$, the $T_{S p}$ values by $5^{\text {th }}$ iteration

are selected as the SSE and Gen (generation) is minimum and optimal.

However, the inconsistency of $S^{3}$ shows that there are two global optimal $X_{i}$ $\left(X_{i}=8187.7 ; 4137.2\right)$, which are frequently appears within the $S B_{0}$ region at $1^{\text {st }}, 2^{\text {nd }}, 4^{\text {th }}, 5^{\text {th }}$, $6^{\text {th }}, 7^{\text {th }}$ and $8^{\text {th }}$ iterations. This has been verified by simulation results in Fig. 4 and 5 of both global optimal $X_{i}$ values of $S^{3}$ and minimum SSE.

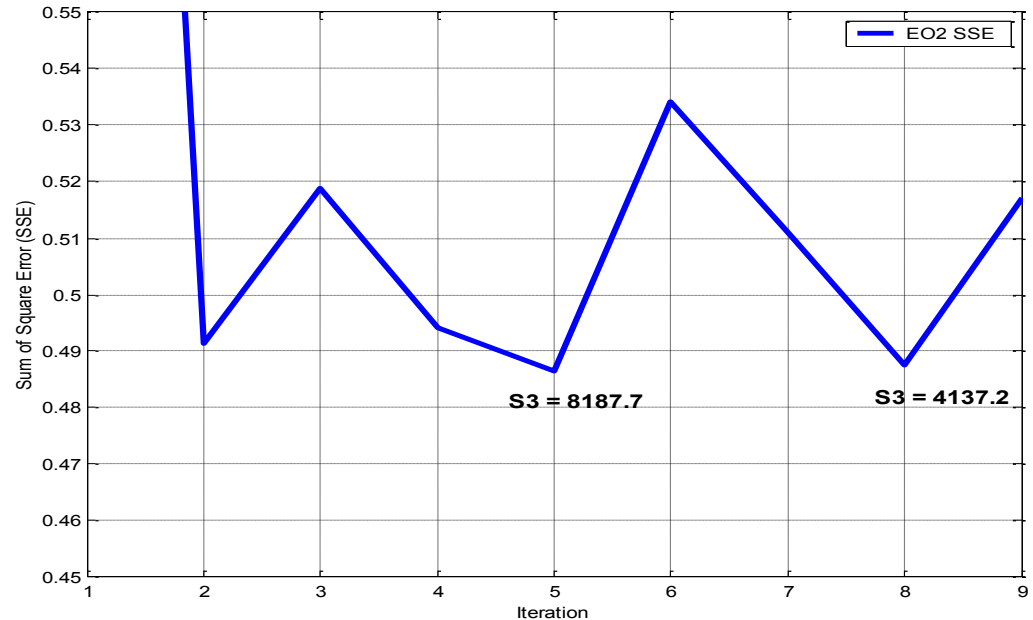

Figure 4: Two optimal values of $S^{3}$ for $\mathrm{EO}_{2}$

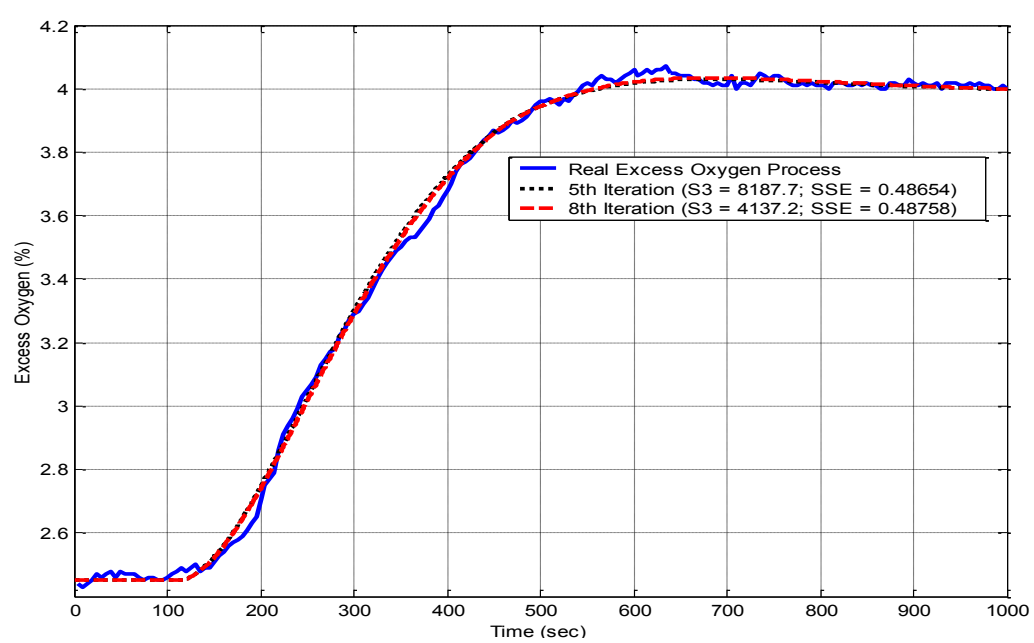

Figure 5: Transient responses of 2 global optimal values with real process of $\mathrm{EO}_{2}$

$$
G(s)_{E O_{2}}=\frac{1.555}{8187.7 s^{3}+14524 s^{2}+181.41 s+1} e^{-109.36 s}
$$




\section{Simulation Results of $3^{\text {rd }}$ Order Transfer Function}

According to the table 2 , the distribution of elite groups within boundary region $\left[X_{i}-\right.$ $\Delta_{G O}, X_{i}+\Delta_{G O}$ ], the exploitation of optimal $X_{i}$ and the consistency of the $T_{S p}$ values of $S^{2}$ and $S^{1}$ in further execution by SGAs are exhibiting similar process characteristics as $\mathrm{EO}_{2}$.

On other hand, the simulation result reveals that the elite group of $T_{S p}$ values of $S^{3}$ are distributed near to $S B_{\text {Lower }}$ region. This is clearly noticeable at $1^{\text {st }}, 2^{\text {nd }}$ and $3^{\text {rd }}$ iterations results that the $T_{S p}$ value of $S^{3}$ is remain exploiting at $S B_{\text {Lower. }}$ This caused the SGAs suffered to exploit an optimal $X_{i}$ and converged to local minima as a part of elite group is located at outside of $S B_{\text {Lower }}$ (state 2). As a result, 3 adjustments on boundaries, especially on $S B_{\text {Lower }}$ are required to optimise the $S B_{O}$ and to bring the elite groups within feasible boundary region. As

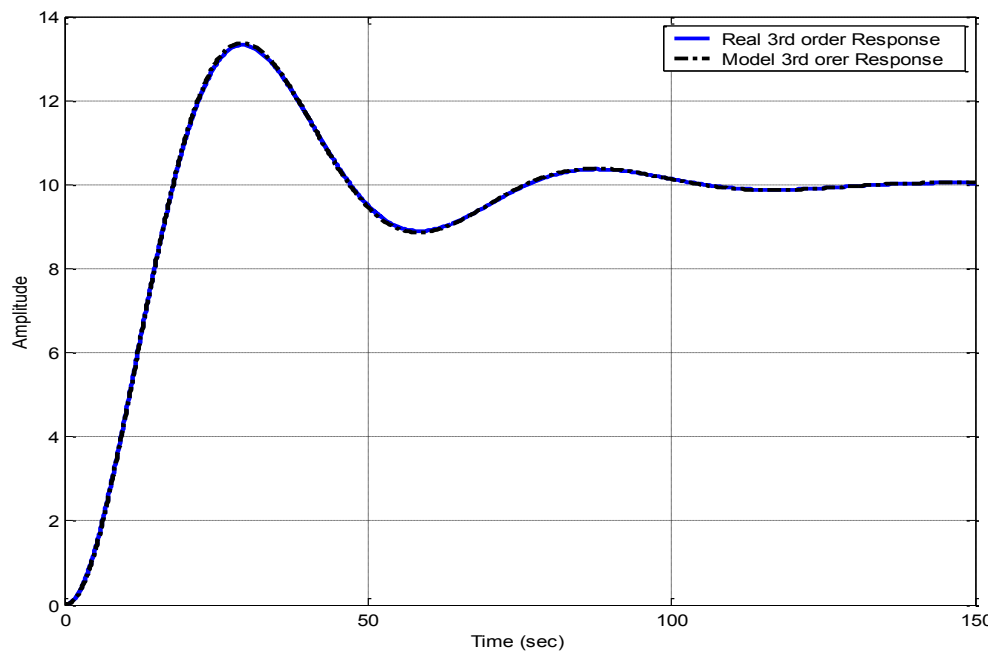

Figure 6: Transient response of $3^{\text {ra }}$ order transfer function real and model process expected, the boundaries are optimised and explored the elite groups well at $4^{\text {th }}$ iteration. Further SGAs execution enhanced an optimal $X_{i}$ exploitation.

Table 2: Simulation Results of $3^{\text {rd }}$ Order Transfer Function Iterations

\begin{tabular}{|c|c|c|c|c|c|c|c|c|c|c|c|}
\hline \multirow{2}{*}{ Iteration } & \multicolumn{2}{|c|}{$s^{3}$} & \multicolumn{2}{|c|}{$S^{2}$} & \multicolumn{2}{|c|}{$s^{1}$} & \multirow{2}{*}{$\begin{array}{l}T s_{p} \\
\left(S^{3}\right)\end{array}$} & \multirow{2}{*}{$\begin{array}{l}T s_{p} \\
\left(S^{2}\right)\end{array}$} & \multirow{2}{*}{$\begin{array}{l}T s_{p} \\
\left(S^{p}\right)\end{array}$} & \multirow{2}{*}{ SSE } & \multirow{2}{*}{ Gen } \\
\hline & $S B_{U}$ & $S B_{L}$ & $S B_{U}$ & $S B_{L}$ & $S B_{U}$ & $S B_{L}$ & & & & & \\
\hline 1 & 29774 & 10 & 3630 & 10 & 98 & 0 & 141.3 & 76.75 & 7.439 & 60.092 & 70 \\
\hline 2 & 280 & 35 & 150 & 20 & & 2 & 42.55 & 77.73 & 6.281 & & 50 \\
\hline 3 & & 12 & & 20 & & 2 & 23.25 & 77.67 & 6.182 & & 30 \\
\hline 4 & 5 & 5 & 15 & 20 & 1 & 2 & 22.98 & 77.69 & 6.179 & & 20 \\
\hline 5 & 50 & 5 & 150 & 20 & 15 & 2 & 21.23 & 77.67 & 6.157 & & 20 \\
\hline 6 & 50 & 5 & 150 & 20 & 15 & 2 & 22.18 & 77.67 & 6.189 & $7.7 \subseteq$ & 30 \\
\hline 7 & 50 & 5 & 150 & 20 & 15 & 2 & 21.98 & 77.68 & 6.197 & 7.6025 & 25 \\
\hline 8 & 50 & 5 & 150 & 20 & 15 & 2 & 21.41 & 77.69 & 6.171 & 7.6171 & 35 \\
\hline 9 & 50 & 5 & 150 & 20 & 15 & 2 & 23.53 & 77.67 & 6.186 & 7.7898 & 25 \\
\hline 10 & 50 & 5 & 150 & 20 & 15 & 2 & 22.62 & 77.68 & 6.175 & 7.7914 & 15 \\
\hline 11 & 50 & 5 & 150 & 20 & 15 & 2 & 23.49 & 77.69 & 6.183 & 7.7895 & 20 \\
\hline
\end{tabular}

Table 3: Simulation Results of $3^{\text {rd }}$ Order Transfer Function with $5 \%$ Disturbance Iterations

\begin{tabular}{|c|c|c|c|c|c|c|c|c|c|c|c|}
\hline \multirow{2}{*}{ Iteration } & \multicolumn{2}{|c|}{$s^{3}$} & \multicolumn{2}{|c|}{$S^{2}$} & \multicolumn{2}{|c|}{$S^{1}$} & \multirow{2}{*}{$\begin{array}{l}T s_{p} \\
\left(S^{3}\right)\end{array}$} & \multirow{2}{*}{$\begin{array}{l}T s_{p} \\
\left(S^{2}\right)\end{array}$} & \multirow{2}{*}{$\begin{array}{l}T s_{p} \\
\left(s^{p}\right)\end{array}$} & \multirow{2}{*}{ SSE } & \multirow{2}{*}{ Gen } \\
\hline & $S B_{U}$ & $S B_{L}$ & $S B_{U}$ & $S B_{L}$ & $S B_{U}$ & $S B_{L}$ & & & & & \\
\hline 1 & 29774 & 10 & 3630 & 10 & 98 & 0 & 380.4 & 82.03 & 11.27 & 150.832 & 90 \\
\hline 2 & 760 & 95 & 165 & 20 & 22 & 3 & 95.15 & 77.78 & 6.296 & 60.1486 & 78 \\
\hline 3 & 190 & 24 & 155 & 20 & 13 & 2 & 25.29 & 77.57 & 6.211 & 33.4558 & 43 \\
\hline 4 & 50 & 6 & 155 & 20 & 13 & 2 & 24.02 & 77.57 & 6.196 & 33.4456 & 37 \\
\hline 5 & 50 & 6 & 155 & 20 & 13 & 2 & 24.67 & 77.58 & 6.049 & 33.4481 & 32 \\
\hline 6 & 50 & 6 & 155 & 20 & 13 & 2 & 24.05 & 76.33 & 6.398 & 33.4452 & 28 \\
\hline 7 & 50 & 6 & 155 & 20 & 13 & 2 & 26.14 & 77.91 & 6.215 & 33.4627 & 22 \\
\hline 8 & 50 & 6 & 155 & 20 & 13 & 2 & 24.25 & 77.51 & 6.198 & 33.4459 & 30 \\
\hline 9 & 50 & 6 & 155 & 20 & 13 & 2 & 22.99 & 77.58 & 6.186 & 33.4503 & 21 \\
\hline 10 & 50 & 6 & 155 & 20 & 13 & 2 & 22.89 & 77.58 & 6.183 & 33.4511 & 42 \\
\hline 11 & 50 & 6 & 155 & 20 & 13 & 2 & 22.76 & 77.84 & 6.114 & 33.4596 & 34 \\
\hline
\end{tabular}


Further, the flexibilities and effectiveness of $T_{S p}$ methods is assessed on $3^{\text {rd }}$ order transfer function model with $5 \%$ disturbance. Initially identified transfer function coefficients without the disturbance are applied on $3^{\text {rd }}$ order model with disturbance. The simulation result in figure 7 reveals that the exploration of elite groups and exploitation of an optimal $X_{i}$ for $3^{\text {rd }}$ order model with disturbance is immensely similar process with $3^{\text {rd }}$ order model without disturbance.

Thus, the effectiveness of $T_{S p}$ methods is well demonstrated in optimizing the $S B_{O}$ and exploiting the $X_{i}$ with or without disturbance. By comparing the identified $T_{S p}$ coefficients with $3^{\text {rd }}$ order transfer function model's coefficients, the $S^{2}$ and $S^{1}$ values have $99 \%$ similarity. But, the $S^{3}$ value only has $54 \%$ of similarity. Nevertheless, the identified model responses, with and without noise, closely match the response of the actual system as illustrated in figure

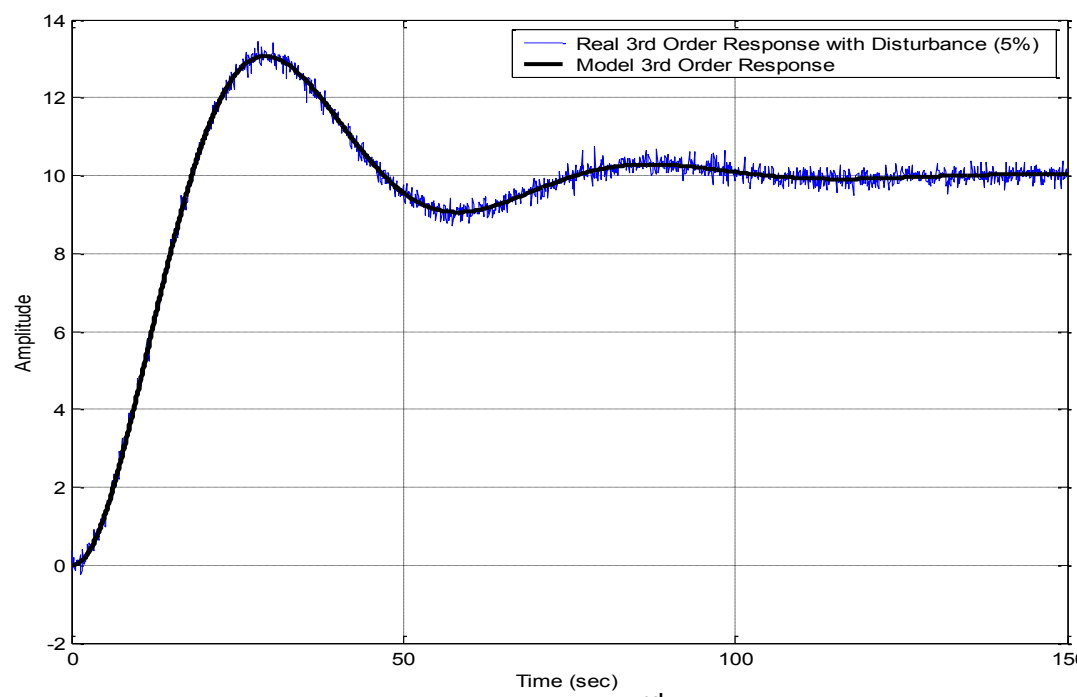

Figure 8: Transient responses of $3^{\text {rd }}$ order transfer function real and model with 5\% disturbance 6 and 7 . Based on minimum SSE, the selected $3^{\text {rd }}$ order model transfer function without disturbance is;

$$
G(s)=\frac{9.997}{21.98 s^{3}+77.68 s^{2}+6.197 s+1}
$$

and with $5 \%$ disturbance is;

$$
G(s)=\frac{9.976}{24.05 s^{3}+76.33 s^{2}+6.398 s+1}
$$

\section{CONCLUSIONS AND FUTURE WORK}

The proposed predetermined time constant $\left(T_{S p}\right)$ method enhanced the optimization of search space boundaries for global optima convergence. The response's dynamic period and settling time provide better presumption of an initial $T_{S p}$ for search space optimisation. The extended $S B_{\text {Upper }}$ and $S B_{\text {Lower }}$ for an optimal search boundary $\left(S B_{O}\right)$ derived from initial $T_{S p}$ brought the elite group within a feasible bounded search region. Further, SGAs execution improved the exploration of elite groups to locate exploit the optimal values for the identified model parameters. As expected, the polynomial coefficients (for $S^{1}, S^{2}$ and $S^{3}$ ) of both $\left(\mathrm{EO}_{2}\right.$ and $3^{\text {rd }}$ order TF) processes are optimised well by SGAs with optimised boundaries. Future work will be carried out on designing the Matlab coding for automatic self adjusting boundary and identification of parameters of more complex models with poles and zeros.

\section{REFERENCES}

[1] R.K. Ursem, "Models for Evolutionary Algorithms and Their Applications in System Identification and Control Optimisation", PhD Thesis, University of Aarhus, Denmark, 2003.

[2] B.F. Zhu, Z.M. Li and B.C. Zhang, "Structural Optimal Design: Theory and Applications", Hydro-Electrical Press, Beijing, China, 1984. 
[3] Z.Y. Wu and Y.T. Wang, "Arch Dam Optimisation Design Under Strength Fuzziness and Fuzzy Safety Measure", Proc. Of Int. Conf. on Arch Dam, Hehai University, Nanjing, China, pp. 129-131, 1992.

[4] Z.Y. Wu and A.R. Simpson, "A Self-Adaptive Boundary Search Genetic Algorithm and its Application to Water Distribution Systems", Journal of Hydraulic Research, Vol. 40, Issue (2), pp. 191-203, 2002.

[5] Y. Lin, J.M. Hao, Z.S. Ji and Y.S. Dai, "A Study of Genetic Algorithm based on Isolation Niche Technique", Journal of Systems Eng., Vol. 15, pp. 86-91, 2000.

[6] Q.Y. Tu and Y.D. Mei, "Comparison of Genetic Simulated Annealing Algorithm and Niche Genetic Algorithm for reservoir Optimal Operation", Hydropower Automation and Dam Monitoring, Vol. 32, pp. 1-4, 2008.

[7] J.L. Jin, X.H. Yang and J. Ding, "An Improved Simple Genetic AlgorithmAccelerating Genetic Algorithm”, Systems Eng. Theory \& Practice, pp. 8-13, 2001.

[8] K.S. Mahendra, R. Gupta and P.R. Bhave, "Optimal Design of Water Networks using Genetic Algorithm with Reduction in Search Space", Journal Water Resource Planning Management, ASCE, Vol. 134 (2), pp. 147-160, 2008.

[9] K. Vairavamoorthy and M. Ali, "Pipe Index Vector: A Method to Improve Genetic Algorithm-Based Pipe Optimisation”, Journal Hydraulic Engineering, ASCE, Vol. 131 (12), pp. 1117-1125, 2005.

[10]B. Xu, P. Zhong and L. Tang, "Improvement on Boundary Searching of Accelerating Genetic Algorithm", Inter. Conf. on Intelligent Design and Engineering Application", pp.301-305, 2012.

\section{ABOUT THE AUTHORS}

K. Rajarathianm, is a PhD student researching Advanced Control Techniques, with the Control Systems Group part of the Mechanical Engineering and Materials Research Centre (MEMARC), School of Engineering, Liverpool John Moores University, UK. E-mail: K.Rajarathinam@2011.ljmu.ac.uk.

Dr. J. Barry Gomm, Reader in Intelligent Systems, Control Systems Group, Mechanical Engineering and Materials Research Centre (MEMARC), School of Engineering, Liverpool John Moores University, UK. E-mail: j.b.gomm@ljmu.ac.uk.

Dr. Karl O. Jones, Principal Lecturer, Control Systems Group, Mechanical Engineering and Materials Research Centre (MEMARC), School of Engineering, Liverpool John Moores University, UK. E-mail: k.o.jones@ljmu.ac.uk.

Prof. DingLi Yu, Professor of Control Systems Engineering, Control Systems Group, Mechanical Engineering and Materials Research Centre (MEMARC), School of Engineering, Liverpool John Moores University, UK. E-mail: d.yu@ljmu.ac.uk. 